\title{
Line Defects at Interfaces in Telluride-Based Thermoelectric Materials
}

\author{
D.L. Medlin*. J.D. Sugar*, N.Heinz**, T. Ikeda**, and G.J. Snyder** \\ * Sandia National Laboratories, 7011 East Avenue, Livermore CA 94551 \\ ** California Institute of Technology, 1200 California Blvd., Pasadena CA 91125
}

Crystallographic line defects, such as steps and dislocations, play important roles in the behavior of interfaces, controlling aspects of interfacial motion, strain accommodation, and morphological development, as well as the functional properties of the interface itself. The analysis of such defects thus provides an important bridge between the atomic-scale structural information that can now be obtained through advanced microscopies and the manifestation of these structural details in terms of macroscopic properties.

In this presentation, I will focus on our recent electron microscopic studies of interfacial line defects in telluride-based thermoelectric materials. I will discuss in particular the solid-state precipitation of tetradymite-structured plates within rocksalt-structured tellurides. Such transformations are of interest because of their potential use in the synthesis of bulk thermoelectric nanocomposites [1]. Previous work on the $\mathrm{AgSbTe}{ }_{2} / \mathrm{Sb}_{2} \mathrm{Te}_{3}[2]$ and $\mathrm{PbTe} / \mathrm{Sb}_{2} \mathrm{Te}_{3}$ [3] systems has shown that the tetradymite structured $\mathrm{Sb}_{2} \mathrm{Te}_{3}$ precipitates in rocksalt telluride matrices form with a plate-like Widmanstätten morphology in which close-packed planes and directions are aligned and the precipitates are elongated parallel to the basal planes of the tetradymite phase. Our HRTEM observations of the $\{111\} \mathrm{AgSbTe}_{2} /(0001) \mathrm{Sb}_{2} \mathrm{Te}_{3}$ interface have identified an interfacial line defect that can mediate the precipitation of these plates [4]. An example of this defect is shown in Figure 1. Our analysis of the geometric properties of this defect - namely its step height and Burgers vectorclarifies the atomic rearrangements involved in the phase transformation. Specifically, motion of this defect accomplishes two functions: (i) to remove a metal plane from the rocksalt matrix, forming a double-chalcogen layer; and (ii) to shear this layer into the correct stacking.

Because this type of defect has both step and dislocation character, it can also play a role in accommodating the interfacial misfit strain. Although the misfit in the $\mathrm{AgSbTe} 2 / \mathrm{Sb}_{2} \mathrm{Te}_{3}$ system is fairly low $(0.8 \%)$, resulting in widely spaced defects, the misfit is an order of magnitude higher for $\mathrm{PbTe} / \mathrm{Sb}_{2} \mathrm{Te}_{3}(6.7 \%)$ resulting in a much higher density of interfacial defects. For instance, Figure 2 shows an array of defects, which are topologically equivalent to the defect shown in Figure 1, along an inclined segment of a $\mathrm{PbTe} / \mathrm{Sb}_{2} \mathrm{Te}_{3}$ interface. The spacing of these defects, which separate short $\{111\} \mathrm{PbTe} /(0001) \mathrm{Sb}_{2} \mathrm{Te}_{3}$ terraces, is consistent with that required to accommodate the interfacial misfit. Moreover, the regular spacing of these defects gives rise to a systematic and predictable inclination away from the $\{111\} /(0001)$ facet plane.

References

[1] D.L. Medlin and G.J. Snyder, Current Opinion in Colloid and Interface Science 14 (2009) 226.

[2] R.W. Armstrong et al., Journal of Appl. Physics. 31 (11) (1960) 1954.

[3] T. Ikeda, V.A. Ravi, G. J. Snyder, Acta Materialia 57 (2009) 666.

[4] D.L. Medlin and J.D. Sugar, Scripta Materialia 62 (2010) 379.

[5] Sandia National Laboratories is a multi-program laboratory managed and operated by Sandia Corporation, a wholly owned subsidiary of Lockheed Martin Corporation, for the U.S. Department of Energy's National Nuclear Security Administration under contract DE-AC04-94AL85000.

Support for this project was provided in part by the Sandia LDRD office. 


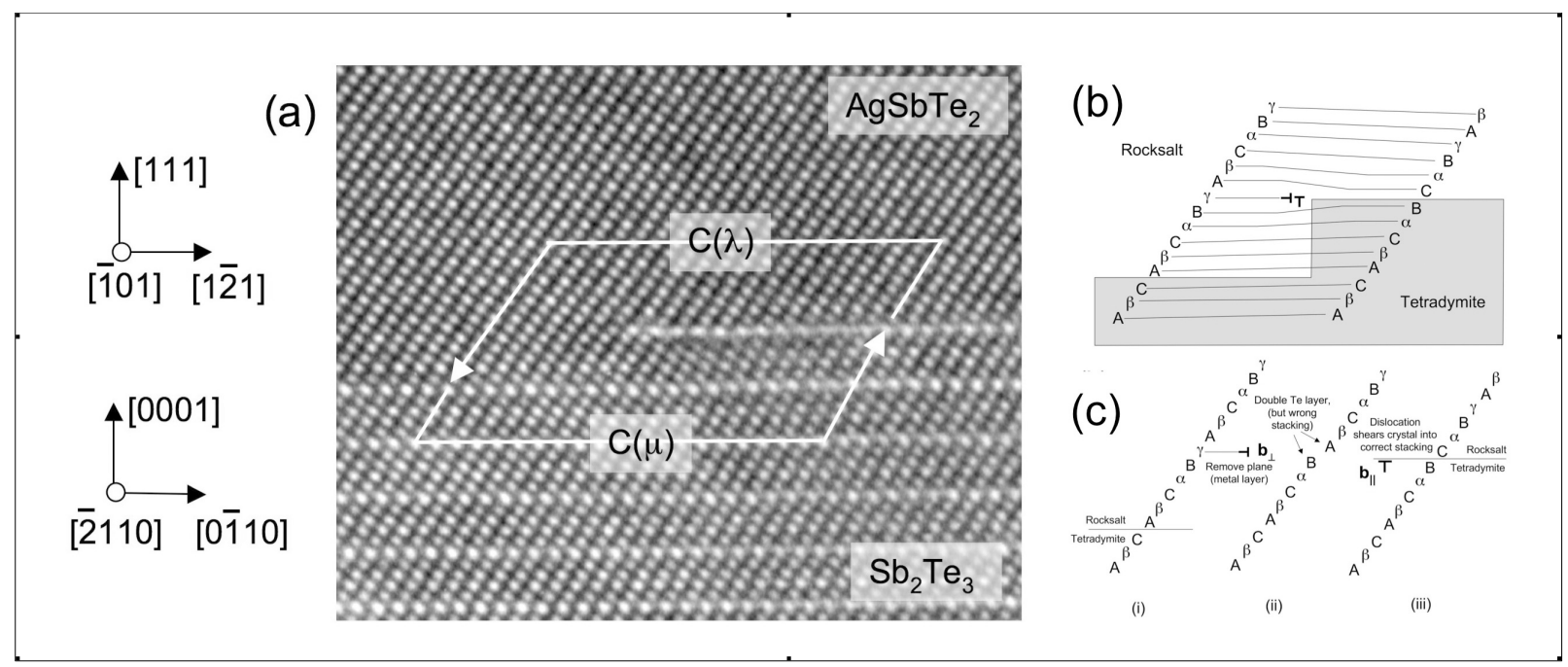

FIG. 1. (a) HRTEM image of an interfacial step defect (or "disconnection) involved in the growth of tetradymite plates in rocksalt tellurides [4]. This defect joins 6 close packed planes in the upper $\mathrm{AgSbTe}{ }_{2}$ crystal to 5 close-packed planes in the lower $\mathrm{Sb}_{2} \mathrm{Te}_{3}$ crystal. For simplicity, we term this a $+6 /+5$ disconnection. As illustrated in (b) and (c), the defect serves two functions in the growth of tetradymite plates: (1) to remove a metal plane (indicated by $\gamma$ ) forming a double chalcogen layer (B A) and (2) to shear the this layer into the correct stacking (BC)

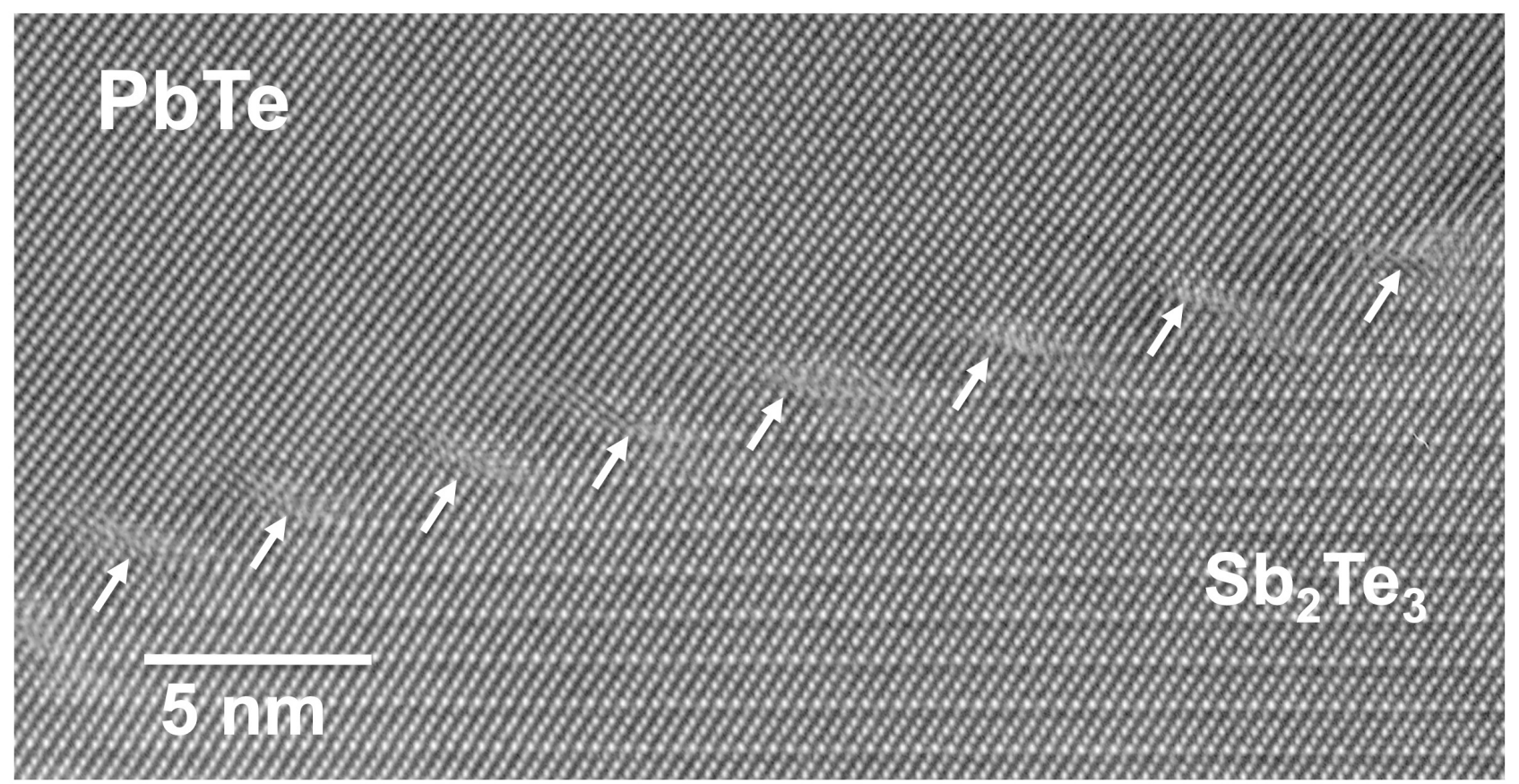

FIG. 2. Array of $+6 /+5$ disconnections at the $\mathrm{PbTe} / \mathrm{Sb}_{2} \mathrm{Te}_{3}$ interface. The large interfacial misfit in this system results in higher densities of interfacial defects than for the $\mathrm{AgSbTe} 2 / \mathrm{Sb}_{2} \mathrm{Te}_{3}$ system. The periodic array results in a systematic departure of the interface inclination from $\{111\} /(0001)$. 